\title{
Research Paper \\ Comparing the Effect of Eight Weeks of Resistance and Endurance Trainings on Physiological and Functional Factors and Record of Elite Runners
}

\author{
Ali Asqar Saberi ${ }^{1}$ (), ${ }^{*}$ Mehrdad Fathi ${ }^{2}$ (), Keyvan Hejazi ${ }^{3}$ ()
}

1. Department of Sport Physiology, Faculty of Sport Sciences, Bojnourd Branch, Islamic Azad University, Bojnourd, Iran.

2. Department of Sport Physiology, Faculty of Sport Sciences, Ferdowsi University of Mashhad, Mashhad, Iran.

3. Department of Sport Physiology, Faculty of Sport Sciences, Hakim Sabzevari University, Sabzevar, Iran.

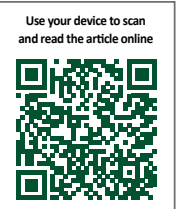

Citation: Saberi A, Fathi M, Hejazi K. [Comparing the Effect of Eight Weeks of Resistance and Endurance Trainings on Physiological and Functional Factors and Record of Elite Runners (Persian)]. Journal of Sport Biomechanics. 2020; 6(1):32-43. https://doi.org/10.32598/biomechanics.6.1.5

https://doi.org/10.32598/biomechanics.6.1.5

Key words:

Blood pressure, Heart rate, Muscle strength

\section{ABSTRACT}

Objective The 3000-meter run is one of the most popular events among endurance and semi-endurance track runnings. Due to its special conditions, aerobic and anaerobic energy production systems can be used at the same time. The aim of the present study was to compare the effect of eight weeks of resistance and endurance trainings on physiological and functional factors and record of elite runners.

Methods In this quasi-experimental study, 22 male elite runners were randomly assigned into the two groups of resistance $(n=11)$ and endurance $(n=11)$ trainings. The resistance and endurance trainings programe was performed for 8 weeks, 3 sessions per week, each for 55-60 minutes. Physiological and functional factors were measured at baseline and at the end of the study. Repeated measures ANOVA was used for within-group and between-group comparisons considering the significant level at $P<0.05$.

Results The training led to significant improvement in heart rate, blood pressure, muscle strength, muscle endurance, balance and 3000-meter record in both groups. There was a significant difference between groups in the mentioned factors $(P<0.05)$.

Conclusion Both resistance and endurance trainings may have a positive effect on runners' records and performance indicators.

\section{Extended Abstract}

\section{Introduction}

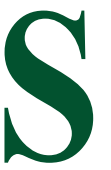

uccess in the athletic performance is related to the several factors, one of which is training [1]. Although training is done with different physical, technical and tactical goals, but in endurance disciplines, especially in athletics, the role of physical and physiological factors is very prominent. On this basis, some endurance instructors believe that people with higher maximal oxygen consumption are able to perform better [1]. The role of these factors is more prominent in trained athletes who have achieved maximum oxygen consumption [2]. The importance of endurance and strength and the relationship between them in sports activities is evident, and when strength and endurance are discussed, physiological and functional issues also arise. The effects of training on the structure and function of the heart depend on the type, intensity and duration of exercise, initial physical fitness, heredity and gender [5].

\section{* Corresponding Author:}

Mehrdad Fathi, PhD.

Address: Department of Sport Physiology, Faculty of Sport Sciences, Ferdowsi University of Mashhad, Mashhad, Iran.

Tel: +98 (51) 38833910

E-mail: mfathei@um.ac.ir 
The effect of resistance training on the endurance has also been studied in the recent years. However, resistance training is not commonly used for endurance runners and it seems that no more than $50 \%$ of the maximum oxygen consumption is included in the strength training [9], and improving maximal oxygen consumption through this type of training is unlikely; however, some evidence suggests that adding resistance training to an aerobic exercise program has a positive effect on the performance of endurance athletes [10].

Due to the lack of research on cardiac adaptation with endurance and resistance training in elite runners and also on the effect of these trainings on physiological and functional factors and the record of elite runners, more study is needed. Therefore, the present study seeks to address the main question of whether the resistance and endurance training programs affect the physiological, functional and record factors of elite runners? And is there a difference between these two training programs? This research can take an effective and small step to identify and solve the problems of runners, and by presenting the results to the community, can help prevent and reduce problems.

\section{Methods}

This is a quasi-experimental study with Pre-test and Post-test design. The study population consisted of all elite male runners in North Khorasan province of Iran who were members of the Bojnourd city athletics team. Among them, 22 volunteers were selected by convenience and purposive sampling methods. First the study objectives and process were explained to them. The samples were then randomly divided into two groups of resistance training $(n=11)$ and endurance training $(\mathrm{n}=11)$. Height of subjects was measured by a stadiometer (Saca, Saca Inc., Germany) with an accuracy of $5 \mathrm{~mm}$, hip and waist circumference by a tape measure (Mabis, Japan) with a sensitivity of $5 \mathrm{~mm}$, and body fat percentage and weight by using Bioelectric impedance device (Inbody, Inbody Inc., South Korea) with a sensitivity of $100 \mathrm{~g}$. Subjects were allowed to enter the study after cardiovascular examination, blood

Table 1. Within-group and between-group comparisons of physiological, functional and record factors

\begin{tabular}{|c|c|c|c|c|c|c|c|}
\hline \multirow{3}{*}{ Factors } & \multirow{3}{*}{ Groups } & \multicolumn{2}{|c|}{ Mean \pm SD } & \multicolumn{4}{|c|}{ Variances } \\
\hline & & \multirow{2}{*}{ Pre-test } & \multirow{2}{*}{ Post-test } & \multicolumn{2}{|c|}{ Within-Group } & \multicolumn{2}{|c|}{ Between-Group } \\
\hline & & & & $\mathbf{t}$ & $\mathbf{P}^{+}$ & $\mathbf{F}$ & $\mathbf{P}$ \\
\hline \multirow[b]{2}{*}{ Heart rate (min) } & Resistance & $72.8 \pm 9.8$ & $137.8 \pm 26.2$ & 0.69 & 0.04 & \multirow[b]{2}{*}{4.661} & \multirow[b]{2}{*}{$0.034^{+}$} \\
\hline & Endurance & $72.3 \pm 9.0$ & $147.7 \pm 24.7$ & 2.23 & 0.04 & & \\
\hline \multirow[b]{2}{*}{ Blood pressure (mm Hg) } & Resistance & $75.43 \pm 7.62$ & $83.92 \pm 2.64$ & 0.15 & 0.007 & \multirow[b]{2}{*}{6.610} & \multirow[b]{2}{*}{$0.012^{+}$} \\
\hline & & $72.57 \pm 12.07$ & $95.07 \pm 9.66$ & 2.20 & & & \\
\hline \multirow{2}{*}{ Aerobic power $(\mathrm{mg} / \mathrm{kg} / \mathrm{min})$} & Resistance & $68.11 \pm 9.60$ & $73.85 \pm 9.00$ & 0.502 & 0.628 & \multirow{2}{*}{0.956} & \multirow{2}{*}{0.332} \\
\hline & Endurance & $65.32 \pm 7.76$ & $69.32 \pm 7.60$ & 0.70 & 0.502 & & \\
\hline \multirow[b]{2}{*}{ Muscle strength (kg) } & Resistance & $12.61 \pm 3.27$ & $13.78 \pm 3.73$ & -1.500 & 0.016 & \multirow[b]{2}{*}{2.544} & \multirow[b]{2}{*}{$0.037^{+}$} \\
\hline & Endurance & $13.51 \pm 3.25$ & $14.65 \pm 3.70$ & -6.091 & 0.001 & & \\
\hline \multirow[b]{2}{*}{ Muscle endurance (kg) } & Resistance & $18.43 \pm 4.15$ & $38.00 \pm 2.36$ & 0.579 & 0.04 & \multirow[b]{2}{*}{42.672} & \multirow[b]{2}{*}{$0.001^{+}$} \\
\hline & Endurance & $17.45 \pm 5.30$ & $41.04 \pm 4.17$ & 3.753 & 0.005 & & \\
\hline \multirow[b]{2}{*}{ Balance (s) } & Resistance & $41.08 \pm 13.52$ & $49.25 \pm 3.25$ & -0.696 & 0.025 & \multirow[b]{2}{*}{0.991} & \multirow[b]{2}{*}{$0.009^{+}$} \\
\hline & Endurance & $45.12 \pm 13.12$ & $52.25 \pm 5.13$ & 0.992 & 0.003 & & \\
\hline \multirow{3}{*}{ 3000-meter record (hs) } & Resistance & $3202 \pm 4.74$ & $2780 \pm 13.12$ & -0.129 & 0.009 & \multirow{3}{*}{4.170} & \multirow{3}{*}{$0.045^{+}$} \\
\hline & & & & & & & \\
\hline & Endurance & $4168 \pm 0.26$ & $3420 \pm 5.13$ & -0.556 & 0.034 & & \\
\hline
\end{tabular}

${ }^{+}$Significant at $\mathrm{P}<0.05$. 
pressure measurement and electrocardiography by a physician.

The Bruce protocol on a treadmill was used to estimate the maximum oxygen consumption. The maximum strength of the subjects was measured using RM1 test according to McGuigan et al. method [18]. To measure the subjects' muscular endurance, McGill's torso muscular endurance test was used in four modes: trunk flexion, trunk extension, right-side bridge, and left-side bridge. Total endurance in the all four directions was considered as final score. To measure the balance, the stork balance test was used according to its standard procedure. This test was performed separately on the dominant and nondominant legs [19]. At the end, the subjects' record was set using the 3000 meters running test. For this purpose, the subject runs 7.5 laps around the 400-meter track.

The training protocol included aerobic (endurance) and resistance trainings for 8 weeks, 3 sessions per week, each for 60 minutes. The aerobic training consisted of running on a treadmill for 21 minutes with intensity equal to $60-70 \%$ of the heart rate reserve. Training intensity was controlled by a heart rate monitor (POLAR, Finland). Pyramid model was used in resistance training. In each session, after 15 minutes of warming up, they performed each movement in 7 sets: 6 repetitions $\times 80 \%$, 3 repetitions $\times 90 \%, 3$ repetitions $\times 90 \%, 3 \times 90 \%$ repetitions, 3 repetitions $\times 90 \%, 3$ repetitions $\times 90 \%$, and 6 repetitions $\times 80 \%$, respectively with a 5 minute interval between each movement. The workouts included leg flexion, leg extension, leg press, squat, lat stretch, chest press, cross-body dumbbell, biceps, triceps, and sit-ups [20].

The collected data were analyzed by SPSS V. 21 software. After ensuring the normality of data distribution by Shapiro-Wilk test and the equality of variances by Levene's test, repeated measures ANOVA was used for within-group and between-group comparisons. Significance level was considered $\mathrm{P}<0.05$.

\section{Results}

The results in Table 1 showed that within-group and between-group differences in heart rate, blood pressure, muscle strength, muscle endurance, balance and 3000-meter record were significant $(\mathrm{P}<0.05)$.

\section{Conclusion}

Runners' heart rate and blood pressure increase after resistance and endurance training, which is due to the du- ration, nature and intensity of trainings with short rest intervals between sessions. It is suggested that these factors of trainings be moderated to reduce the muscle fatigue of runners.

\section{Ethical Considerations}

\section{Compliance with ethical guidelines}

Prior to study, a written informed consent was signed by the participants after explaining the study objectives and methods. They were assured of the confidentiality of their information, and were free to leave the study at any time.

\section{Funding}

This research did not receive any specific grant from funding agencies in the public, commercial, or not-forprofit sectors.

\section{Authors' contributions}

Implementation: Ali Asghar Saberi, Mehrdad Fathi; Final review of the manuscript: All authors; Conceptualization: Mehrdad Fathi; Data analysis: Keyvan Hejazi; Performing the study and compiling the manuscript: Keyvan Hejazi.

\section{Conflicts of interest}

The authors declare no conflict of interest. 


\title{
مقايسه تأثير هشت هفته تمرين مقاومتى و استقامتى بر فاكتورهاى فيزيولوزيكى، عملكردى و ركورد دوندكان نخبه
}

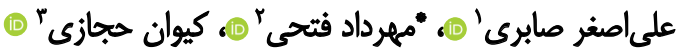 \\ ا. كروه فيزيولورى ورزشى، دانشكده علوم ورزشى، دانشَّاه آزاد اسلامى واحد بجنورد، بجنورده، ايران.

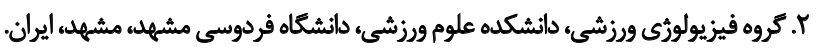

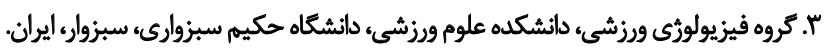

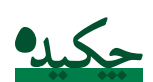

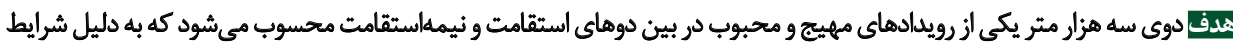

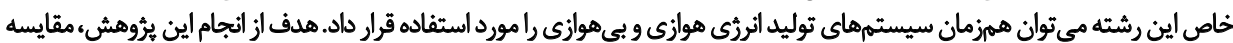

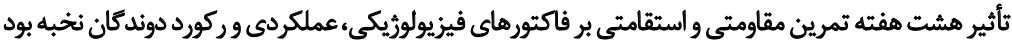

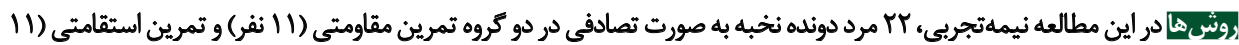

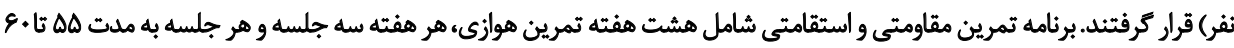

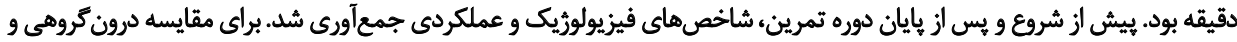

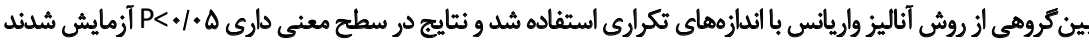

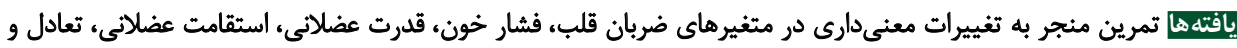

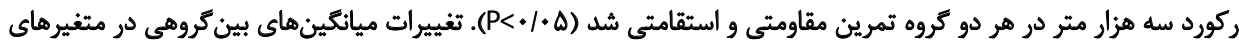

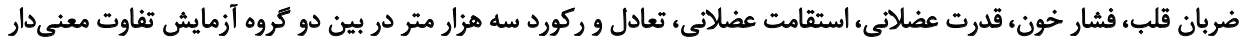

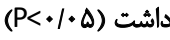

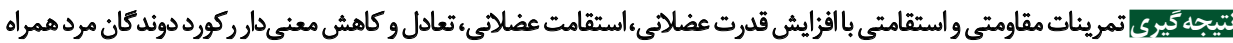

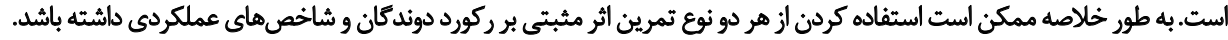

اطلاعات مقاله:

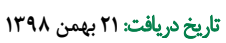

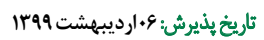
تاريخ انتشار: rاخرداد

كليدوازوها: فشار خون، ضربان قلب،

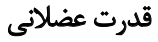

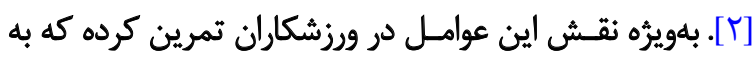

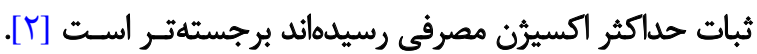

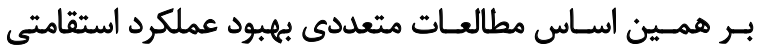
و عوامل تأثيركذار بر آن را به دنبال تمرينات مختلف كزاري

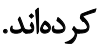

بنابراين بخش عمدهاي از تفاوتهاى فيزيولوريكى در عملكرد

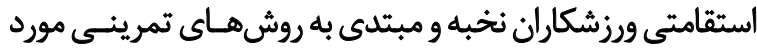

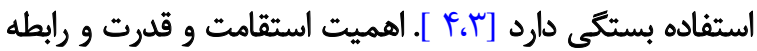

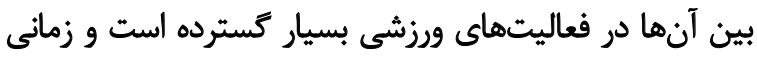

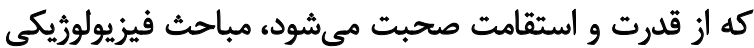
و عملكردى نيز به ميان مى آيد. آثار ورزش بر بر ساختار و و عملكرد

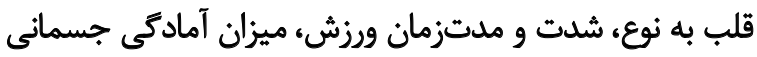

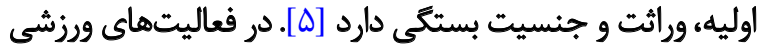
كه به طور ديناميك انجام مى شوند، فشار خون سيستولى در حد

موفقيت در عملكرد ورزشى با عوامل متعددى در ارتباط است

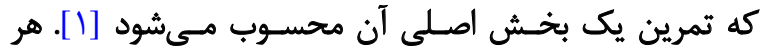

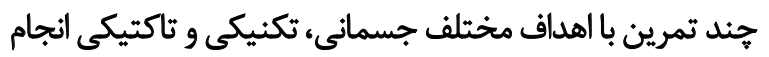

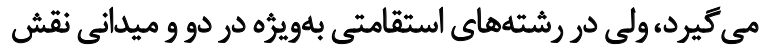

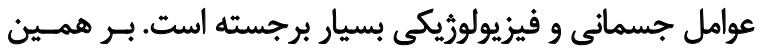

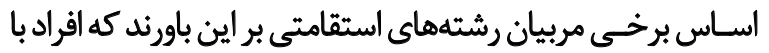

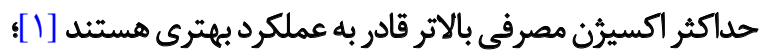

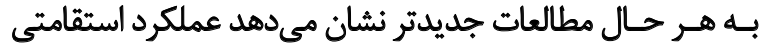

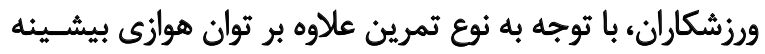

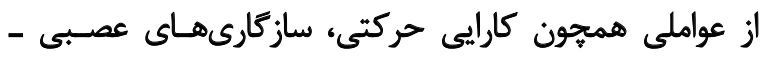

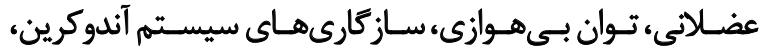
آستانه لاكتات و توانايى به تأخير انداختن آن تأثير مىيذئيرد آندين 
بنابراين شايد استفاده از تمرينات مقاومتى در كنار تمرينات

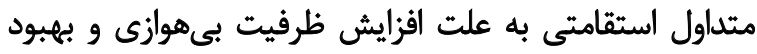

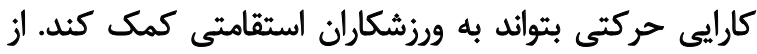

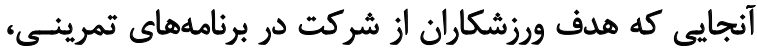

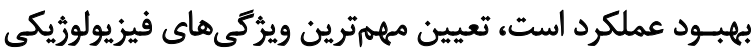

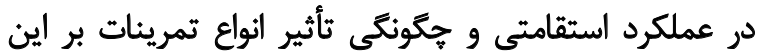

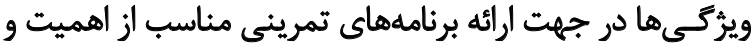

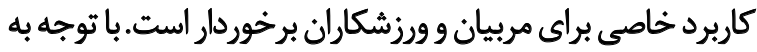

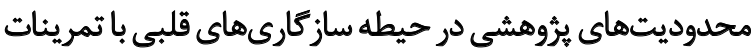

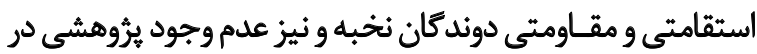

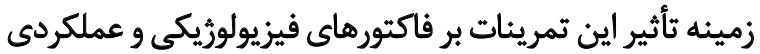

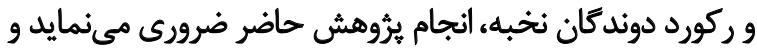

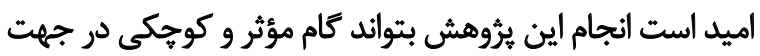

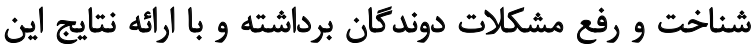

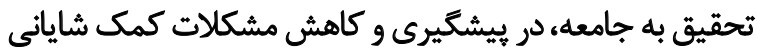

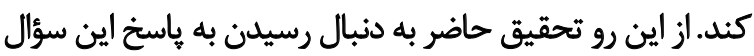

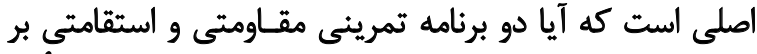

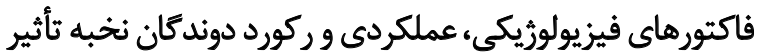

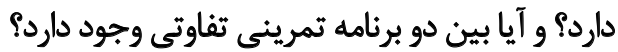

$$
\text { روشّشئاسي }
$$

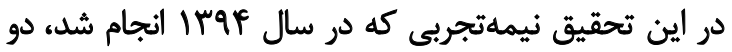

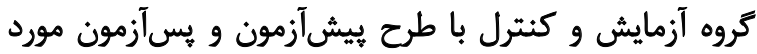

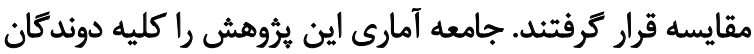

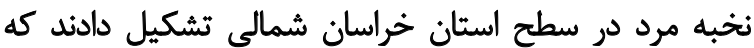

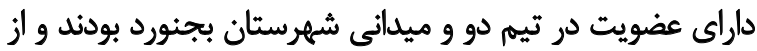

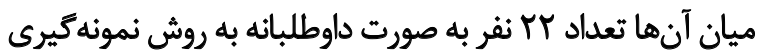

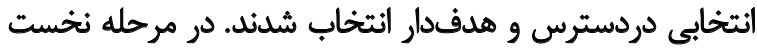

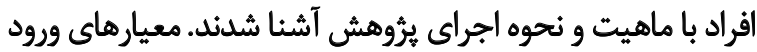

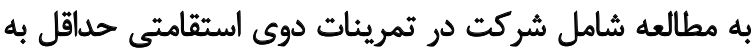

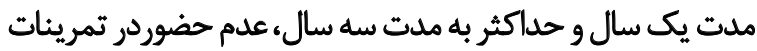
مـنظم تداومى و تناوبى هوازى، عدم ابتلا به بيمارى و و عارضه

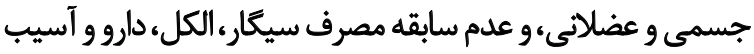

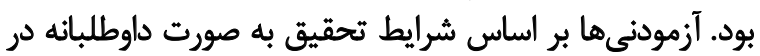

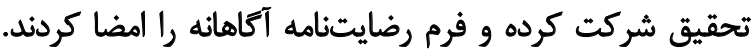

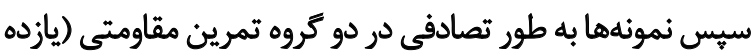
نفر) و تمرين استقامتى (يازده نفر) دستهبندى شرون شديند.

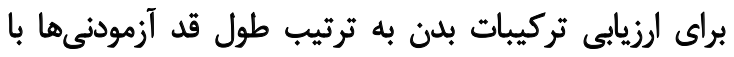

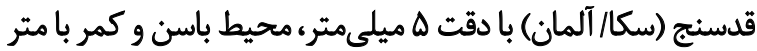

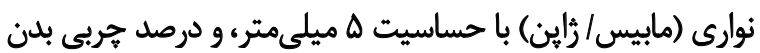

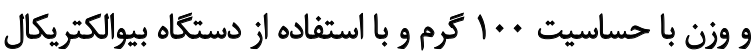

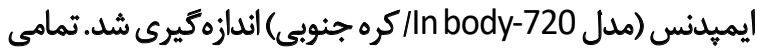

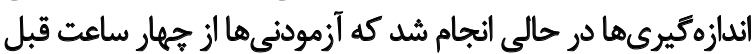

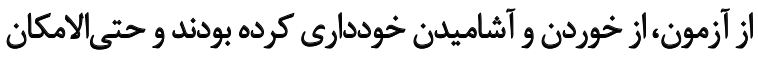

قابل ملاحظه افزايش يُيدا مي كند، در حالى كه فشار دياستولى عالى

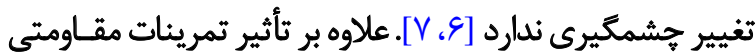

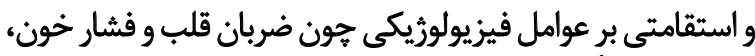

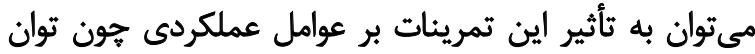

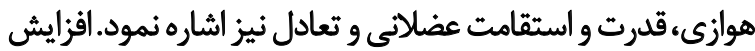

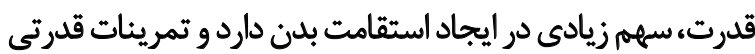
و استقامتى لازم و ملزوم يكديكر هستند [1]]. اثر تمرينـات مقــاومتى بـر عملكرد اسـتقامتى نيز موضوعيى

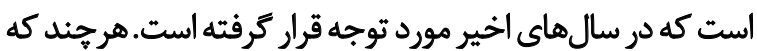

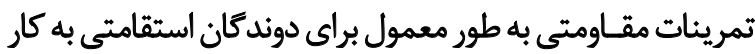

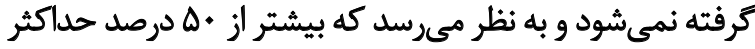

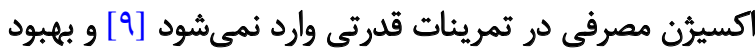

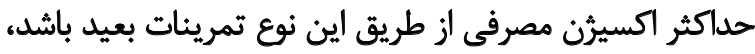

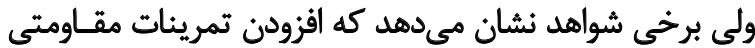

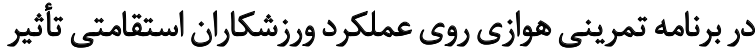

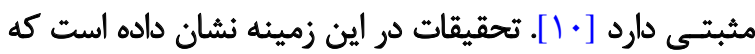

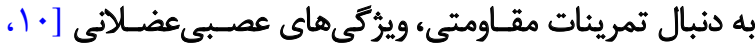

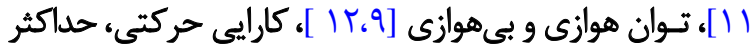

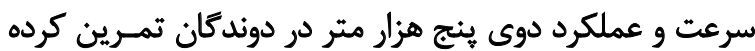

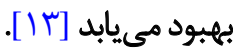

براندون تزارش كرد دوندههـايى كه از نظـر حداكثر اكسيرن

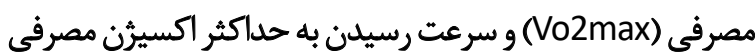

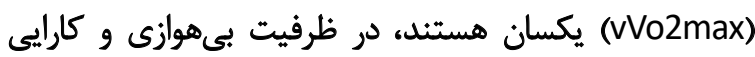

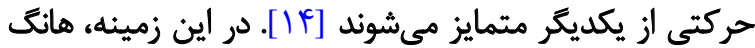

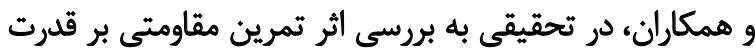

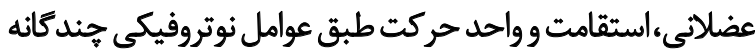

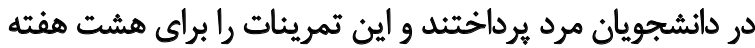

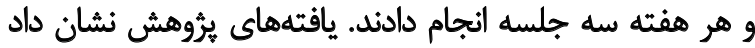

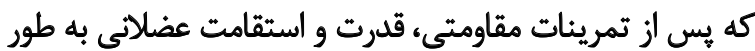

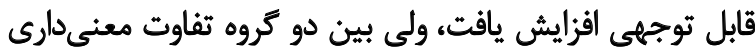

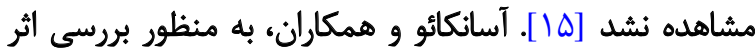

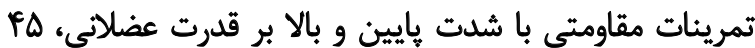

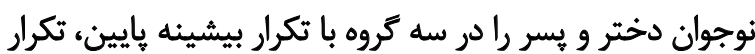

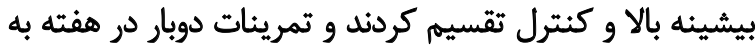

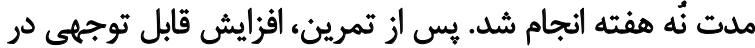

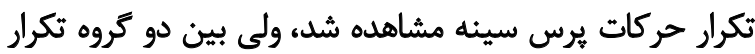

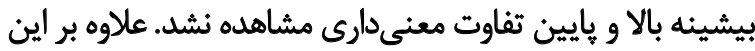

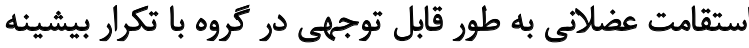

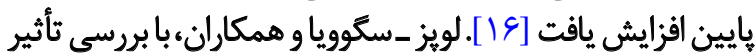

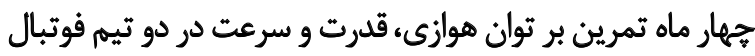

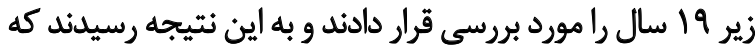

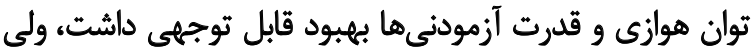

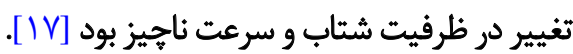




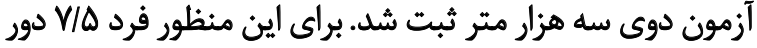

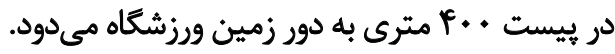
هروتكل تمرينى شامل تمرينات هوازى (استقامتى) همراه

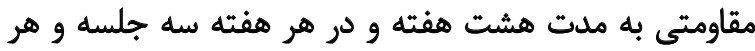

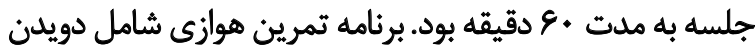

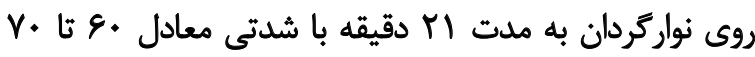

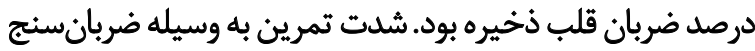

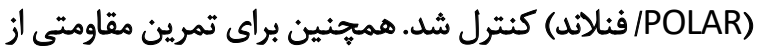

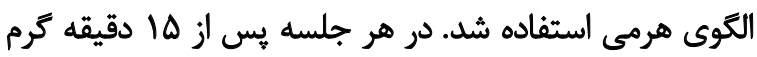

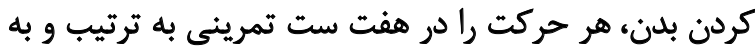

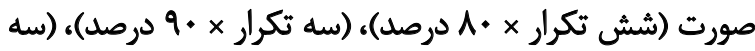

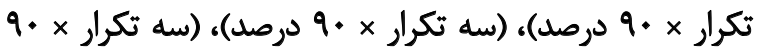

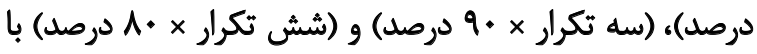

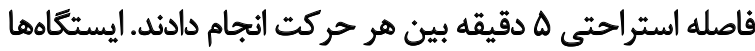

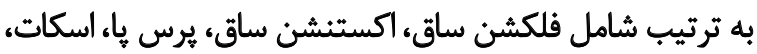

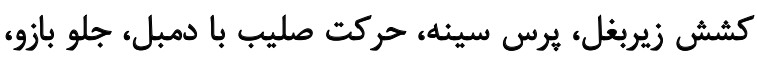

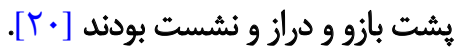
دادههاى جمعآورىشده با نرمافزار SPSS نسخه I آ تجزيه

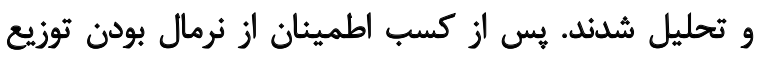

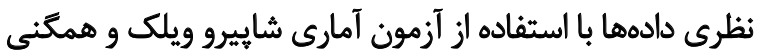

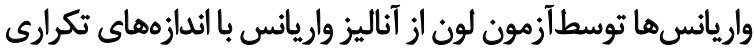

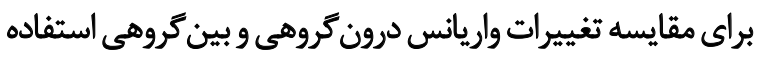

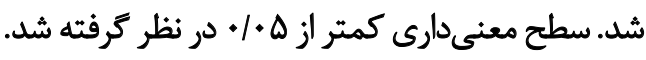

نثايج

مشخصات آزمودنىهاي كروه آزمايش و كنترل در جدول شماره آ آورده شده است آمودئ.

نتايج جدول شماره r نشان مسي دهد كه تغييرات ميانكينهاي

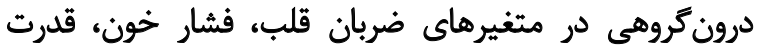

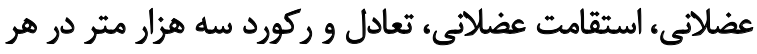

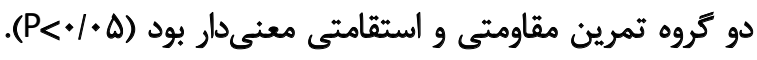

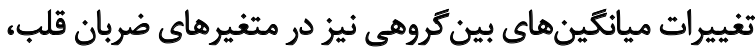

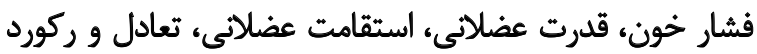

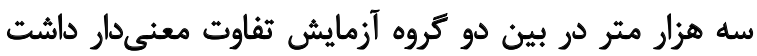

. (P< $(P \circ \bullet)$

H

هدف از مطالعه حاضر بررسى تأثير هشت هفته تمرين

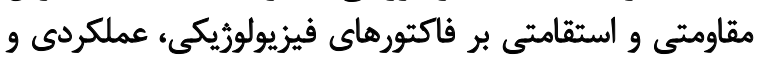

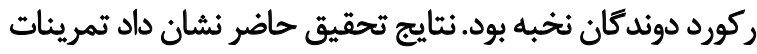

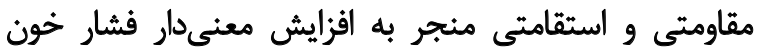

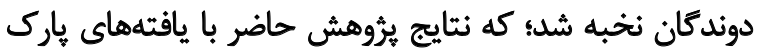

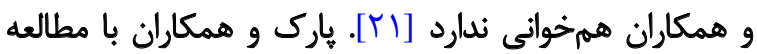

مثانه، معله و روده آنها تخليه شده بود. آزمودنى ها يس ازئ معاينه

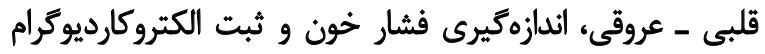
توسط يزشك متخصص، مجوز ورود به طرح را كسب كردئ فرند.

ميزان فشار خون هرآزمودنى قبل از شروع فعاليت بدنى با بانيا

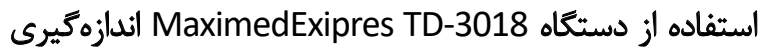

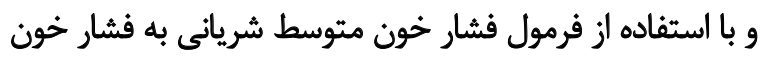
متوسط تبديل شد (فرمول شماره ():

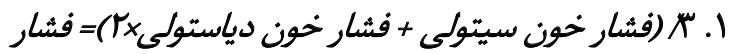
خون متوسط

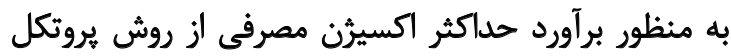

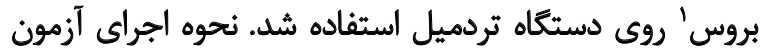

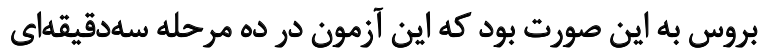

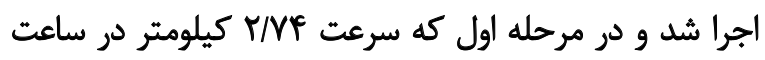

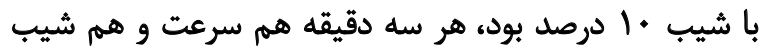

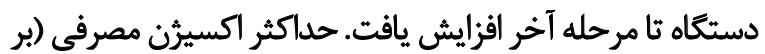

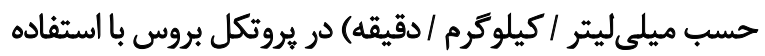

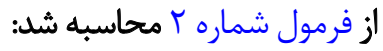

Tr اكسيُّن مصرفى

قدرت بيشينه آزمودنى ها با استفاده از آزمون 1RM (يك تكرار

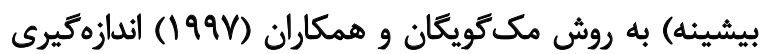

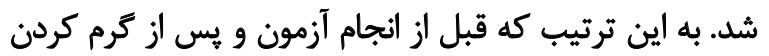

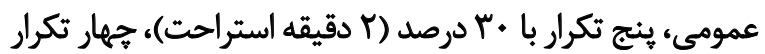

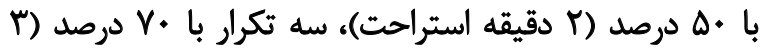

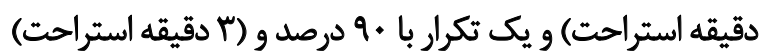

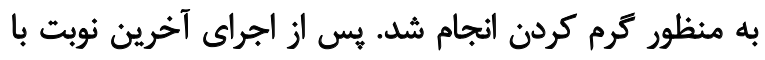

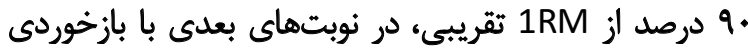

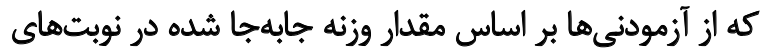

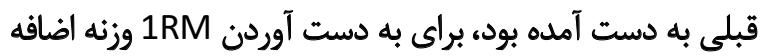

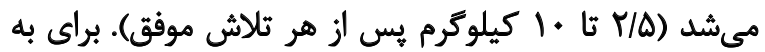

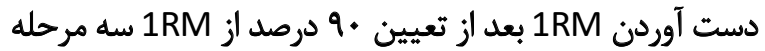

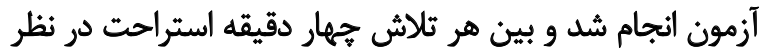

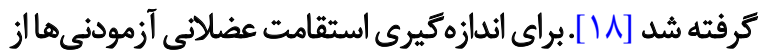

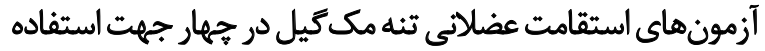

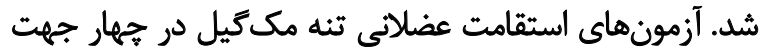

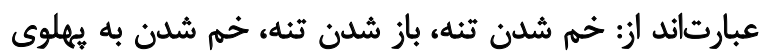

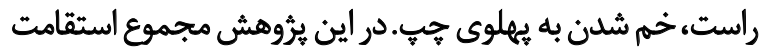

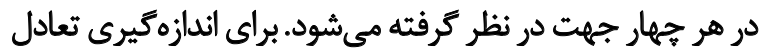

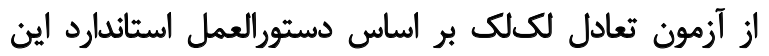

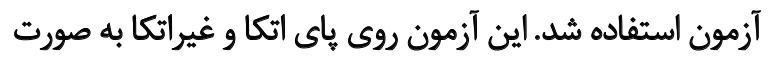

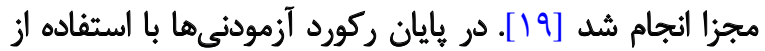

1. Borus 
جدول ا. ويثگى هاى آنترويومتريك بيماران همودياليزى شركتكنيده در مطالعه

\begin{tabular}{|c|c|c|c|c|}
\hline \multicolumn{4}{|c|}{ مياتكين +انحراف استاندارد } & \multirow[b]{2}{*}{ كروهها } \\
\hline نمايه توده بدن (كيلوكرم / مترميع) & وزن (كيلوكرم) & قد (سانتىمتر) & سن (سال) & \\
\hline$r \cdot / r \pm \pm r / a r$ & $9 / / \% 9 \pm 1.1+0$ & $|V N \cdot| \pm \varphi \mid+1$ & $M / q \cdot \pm 1 / M$ & مقاومتى (Iانفر) \\
\hline$r / / . \pm \pm / \Delta r$ & $\varepsilon q / \Delta Y \pm N F \Delta$ & $\mid A Y / \cdot r \pm \varphi / \cdot r$ & $r m / 1 \cdot \pm V / \Delta q$ & استقامتى (Iانفر) \\
\hline
\end{tabular}

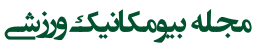

جدول r. مقايسه تغييرات واريانس درون كروهى و بين كروهى در شاخص هاى فيزيولوزيكى، عملكردى و ركورد دوندكان نخبه

\begin{tabular}{|c|c|c|c|c|c|c|c|}
\hline \multicolumn{4}{|c|}{ تغييرات } & \multicolumn{2}{|c|}{ ميانكيند اندحراف استاندارد } & \multirow{3}{*}{$\log _{0}$} & \multirow{3}{*}{ متغيرها } \\
\hline \multicolumn{2}{|c|}{ يبن كروه } & \multicolumn{2}{|c|}{ دوون كروه } & \multirow[b]{2}{*}{ يسأزمون } & \multirow[b]{2}{*}{ ي يشآزمون } & & \\
\hline معنى مارى & $\mathbf{F}$ & معنى سطاري & $\mathbf{t}$ & & & & \\
\hline \multirow{2}{*}{$.1 . m e t$} & \multirow[t]{2}{*}{ flegl } & $.1 \cdot \varphi^{+}$ & .199 & & & & \multirow{2}{*}{ ضربان قلب (دقيقه) } \\
\hline & & $.1 \cdot \varphi^{\dagger}$ & $r / \pi r$ & IPV/VETE/V & $n / w \pm V$ & اسثقامتى & \\
\hline \multirow{2}{*}{$.1 \cdot 1 r^{+}$} & \multirow{2}{*}{$9(8)}$. & & & & & & \multirow{2}{*}{ (ميلىمثر جيوه) } \\
\hline & & $\cdot 1 * \mathrm{~N}^{+}$ & $r / r$. & $৭ \Delta / \cdot \bigvee \pm \vee / \&$ & $V / \Delta V \pm \mid Y / \cdot V$ & استقامتى & \\
\hline \multirow{2}{*}{. MTM } & \multirow{2}{*}{$.19 \Delta 8$} & . IETA & $\cdot / \Delta \cdot r$ & $\gamma / \Lambda \Delta \pm V / .$. & SNIIIV/ & مثقاومتى & \multirow{2}{*}{ 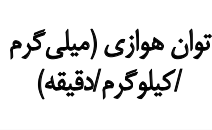 } \\
\hline & & $+10+r$ & $\cdot N$ & $\varepsilon q / H \pm V V / \varepsilon$. & EQ/TTIVIVE & استقامتى & \\
\hline \multirow[t]{2}{*}{$.1 \cdot r^{+}$} & \multirow{2}{*}{ T/APF } &.$/ \cdot 18^{\dagger}$ & $-1 / \Delta \ldots$ & $1 \Psi / V \lambda \pm r / N$ & & مقاومتى & \multirow{2}{*}{ قدرت عضلانى } \\
\hline & &.$* 1^{+}$ & $-8 / .91$ & $\mid r / \varepsilon \Delta \pm \Psi / V$ & $|\Psi / \Delta| \pm T / K \Delta$ & استقامتى & \\
\hline \multirow[t]{2}{*}{$+1+* 1^{\dagger}$} & \multirow[t]{2}{*}{ rr/ent } & $.10 p^{t}$ & & & & مقاومتى & \multirow{2}{*}{ 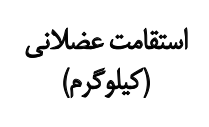 } \\
\hline & & $\cdots / \cdots \Delta^{\dagger}$ & renar & $P V \cdot \bullet \pm+/ / V$ & $\mid V / T \Delta \pm \Delta / T^{\mu}$ & استقامتى & \\
\hline \multirow{2}{*}{$.1+.9^{+}$} & \multirow{2}{*}{.$/ 991$} &.$/ \cdot Y \Delta^{\dagger}$ &.$- / 8 q 8$ & $P Q / T \Delta \pm r / T \Delta$ & $P V / \cdot \lambda \pm I r / \Delta T$ & 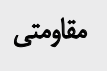 & \multirow{2}{*}{ تعادل } \\
\hline & & $.1 . \cdot \mu^{+}$ &.$/ 997$ & $\Delta T / T \Delta \pm \Delta / / T$ & $P A / / T \pm I T / N$ & استقامتى & \\
\hline \multirow{2}{*}{$.1 \cdot F \Delta^{+}$} & \multirow{2}{*}{ elir. } & $.1+.9^{\dagger}$ & $-\infty / 1 p q$ & $r V A+ \pm I r / I r$ & $M \cdot Y \pm Y / M^{C}$ & مثقاومتى & \multirow{2}{*}{ ركورد (سله هزار متر) } \\
\hline & & $\cdot / \cdot m e^{\dagger}$ & $-+/ \Delta \Delta F$ & $M T \cdot \pm \Delta / / T$ & FIFNt./TE & استقامتى & \\
\hline
\end{tabular}

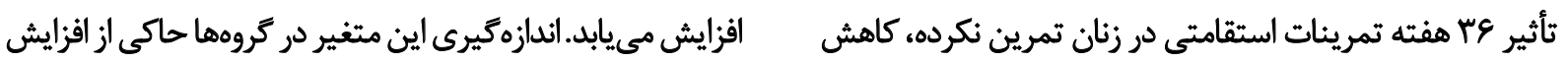

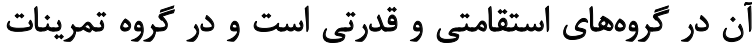

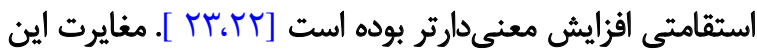

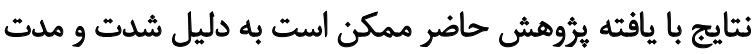

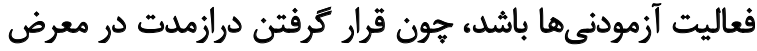

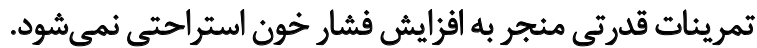
نتايج تحقيق حاضر نشان داد تمرينات مقاومتى و استقامتى دئى

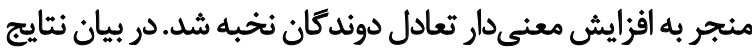

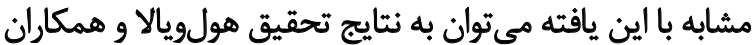

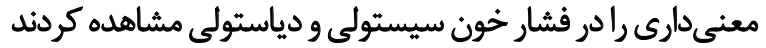

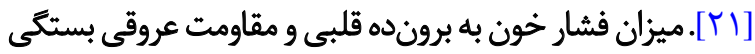

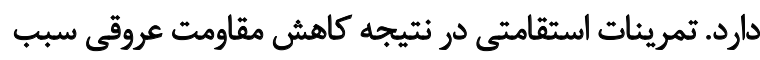

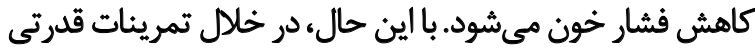

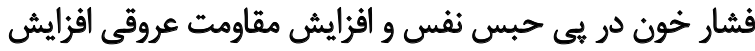

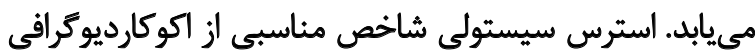

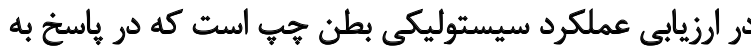
افزايش بسيار و مقاومت عروقى ناشى از انجام تمرينات قدرتى 
نتايج تحقيق حاضر نشان داد تمرينات مقاومتى و استقامتى

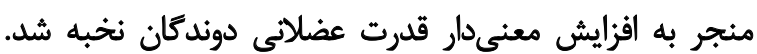

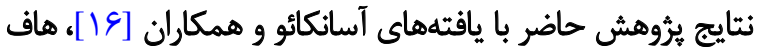

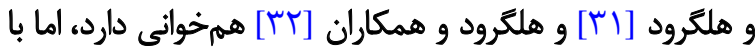

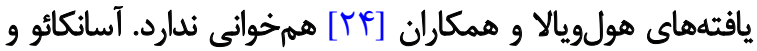

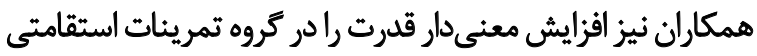

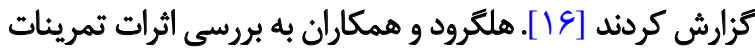

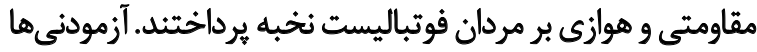

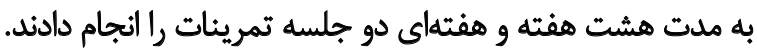

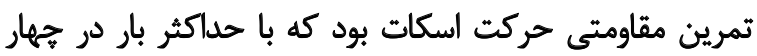

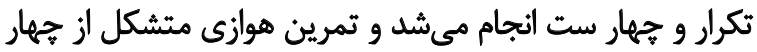

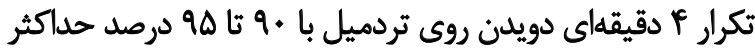

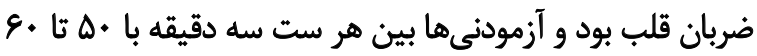

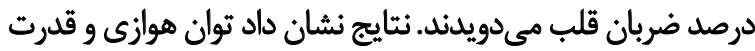

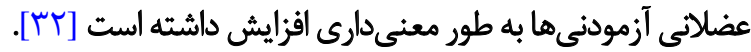

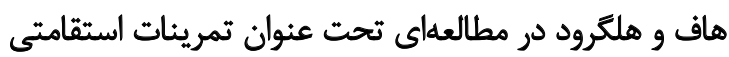

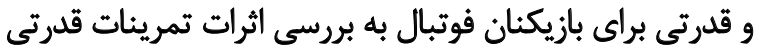

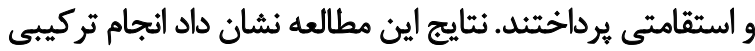

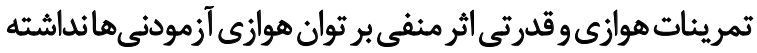

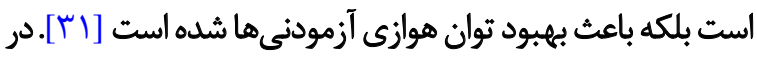

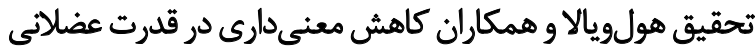

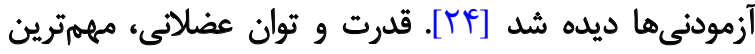

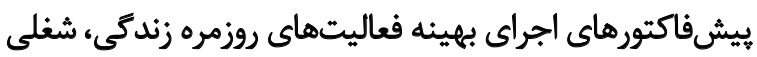

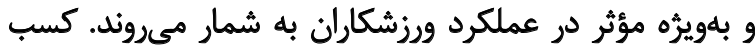

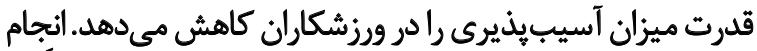

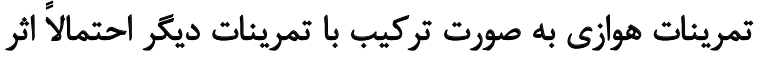

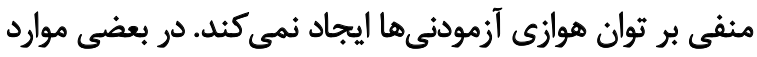

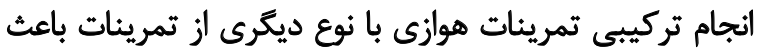

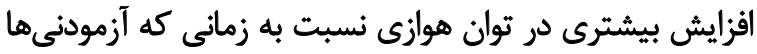

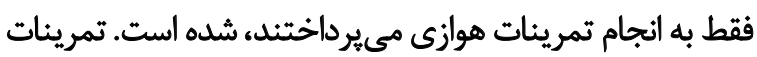

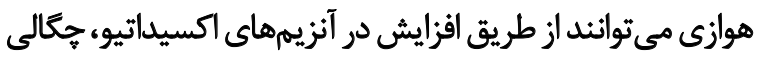

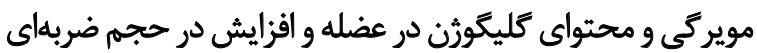

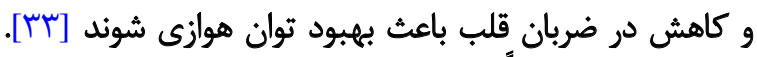

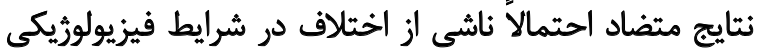

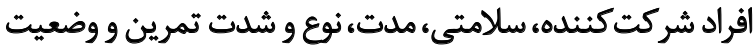

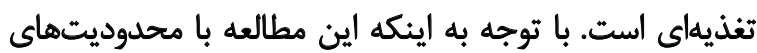

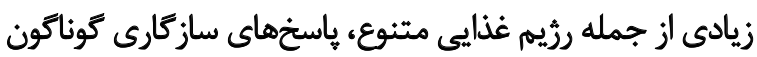

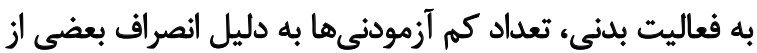

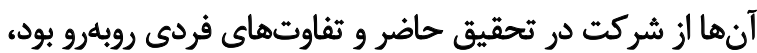

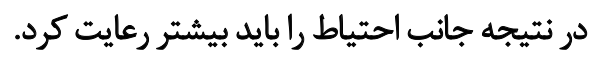

\section{نتيجهيَيرى نهايى}

نتايج يُروهش حاضر نشان داد كه ضربان قلب و فشار خون

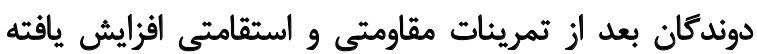

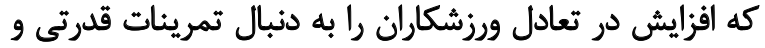

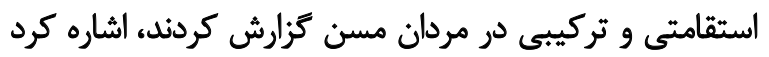

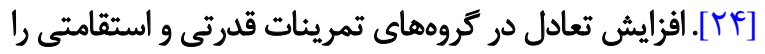

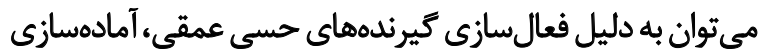

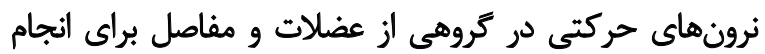

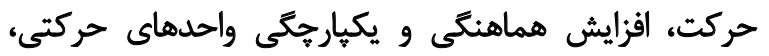

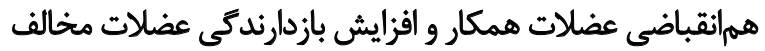

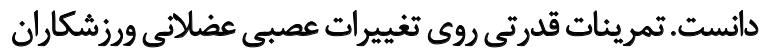

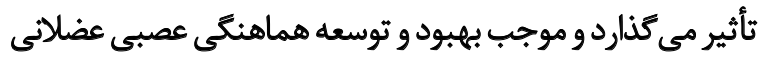

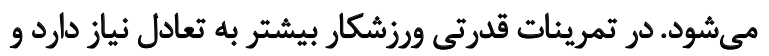

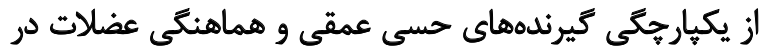

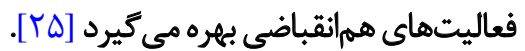

نتايج تحقيق حاضر نشان داد تمرينات مقاومتى و استقامتى

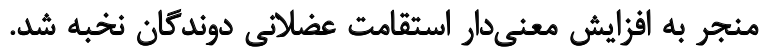

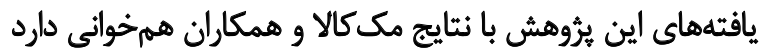

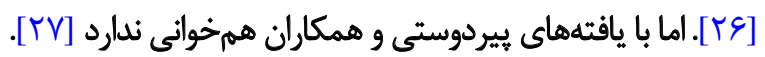

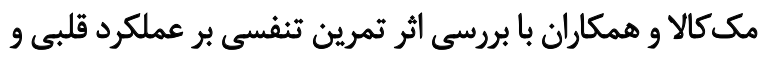

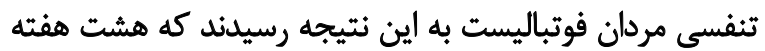

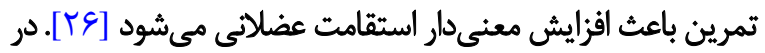

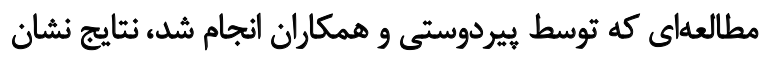

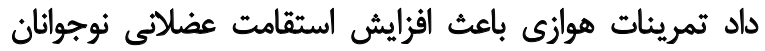

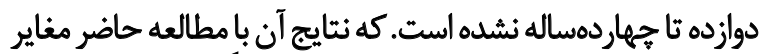

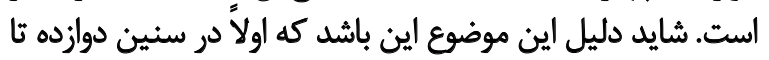

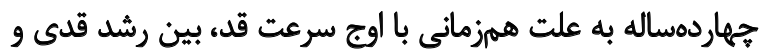
افزايش قدرت عضلانى تعادلى وجود ندارد [T/ [TV].

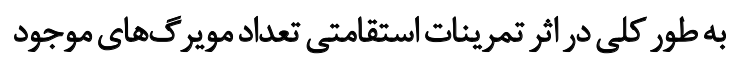

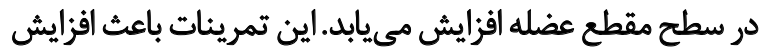

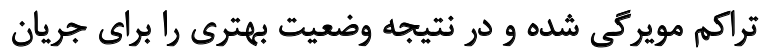

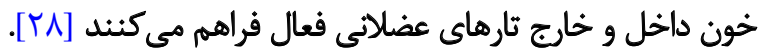

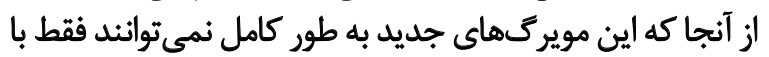

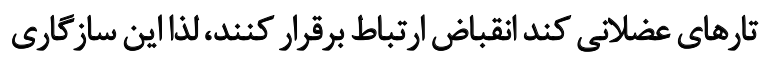

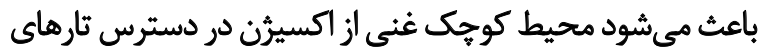

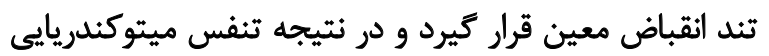

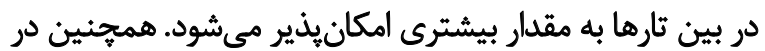

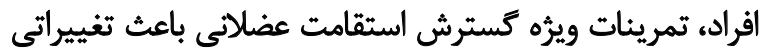

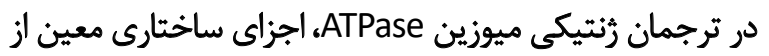

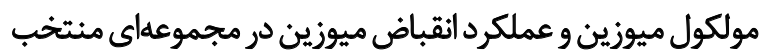

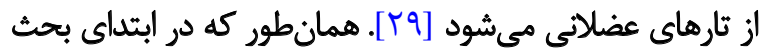

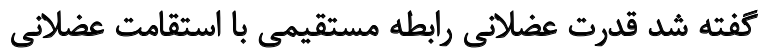

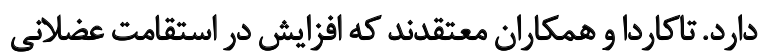

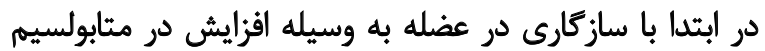

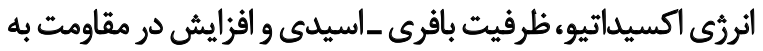

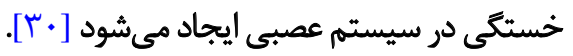


است كه علت اين افزايش به دليل طول مدت تمرينات، ماهيت

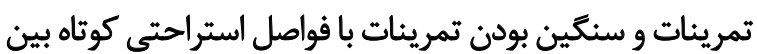

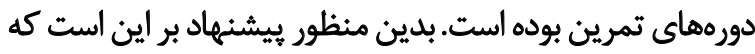

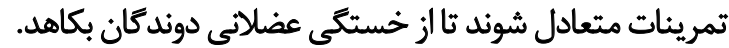

\section{مالاحظات اخلاقى}

$$
\text { يبيروى أز اصول اخلاق بثوهش }
$$

به تمام آزمودنى ها فرم رضايتنامه شركت در يثروهش داده شد إند

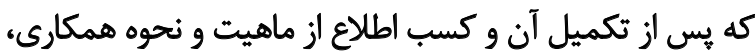

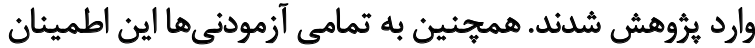

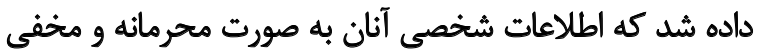

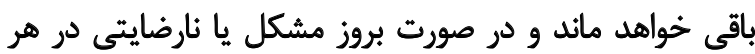

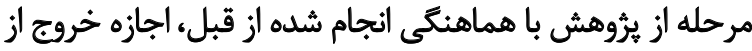

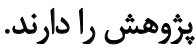

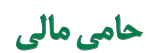

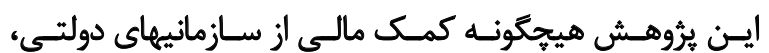

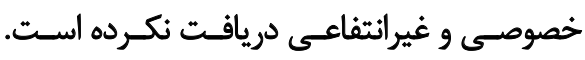

$$
\text { مشاركت نويسند مثان }
$$

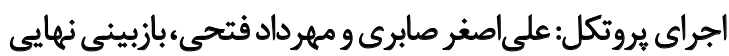

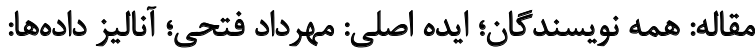
كيوان حجازى؛ اجراى مطالعه و تدوين دستنوشته: كيوان ايدان

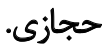

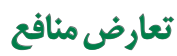

بنابر اظهار نويسندكان، اين مقاله تعارض منافع ندارد. 


\section{References}

[1] Kenefick RW, Mattern CO, Mahood NV, Quinn TJ. Physiological variables at lactate threshold under-represent cycling time-trial intensity. J Sports Med Phys Fitness. 2002; 42(4):396-402. [PMID]

[2] Coyle EF. Physiological determinants of endurance exercise performance. J Sci Med Sport. 1999; 2(3):181-9. [DOI:10.1016/S14402440(99)80172-8

[3] Mujika I, Goya A, Ruiz E, Grijalba A, Santisteban J, Padilla S. Physiological and performance responses to a 6-day taper in middle-distance runners: influence of training frequency. Int J Sports Med. 2002; 23(5):36773. [DOI:10.1055/s-2002-33146] [PMID]

[4] Evans SL, Davy KP, Stevenson ET, Seals DR. Physiological determinants of $10-\mathrm{km}$ performance in highly trained female runners of different ages. J Appl Physiol. 1995; 78(5):1931-41. [DOI:10.1152/jappl.1995.78.5.1931] [PMID]

[5] Jerry J, Moya M, Kravitz LA. A review of the acute cardiovascular responses to resistance exercise of healthy young and adults. J Strength Cond Res. 1999; 13(1):90-6. [DOI:10.1519/00124278-19990200000016]

[6] Langdeau JB, Blier L, Turcotte H, O'Hara G, Boulet LP. Electrocardiographic findings in athletes: the prevalence of left ventricular hypertrophy and conduction defects. Can J Cardiol. 2001; 17(6):655-9. [PMID]

[7] Vinereanu D, Florescu N, Sculthorpe N, Tweddel AC, Stephens MR, Fraser AG. Left ventricular long-axis diastolic function is augmented in the hearts of endurance-trained compared with strength-trained athletes. Clin Sci. 2002; 103(3):249-57. [DOI:10.1042/cs1030249] [PMID]

[8] Wilmore JH, Costill DL. Physiology of sport and exercise. Champaign Human kinetics; 1994. https://books.google.com/books/about/Physiology_of_Sport_and_Exercise.html?id=ABdrAAAAMAA.

[9] Jung AP. The impact of resistance training on distance running performance. Sports Med. 2003; 33(7):539-52. [DOI:10.2165/00007256200333070-00005] [PMID]

[10] Creer AR, Ricard MD, Conlee RK, Hoyt GL, Parcell AC. Neural, metabolic, and performance adaptations to four weeks of high intensity sprintinterval training in trained cyclists. Int J Sports Med. 2004; 25(2):92-8 [DOI:10.1055/s-2004-819945] [PMID]

[11] Paavolainen L. Neuromuscular characteristics and muscle power as determinants of running performance in endurance athletes: With special reference to explosive-strength training. Finland: University of Jyväskylä; 1999. https://books.google.com/books/about/Neuromuscular_Characteristics_and_Muscle.html?id=_QhZAAAAYAJ

[12] Marcinik EJ, Potts J, Schlabach G, Will S, Dawson P, Hurley BF. Effects of strength training on lactate threshold and endurance performance. Med Sci Sports Exerc. 1991; 23(6):739-43. [DOI:10.1249/00005768 199106000-00014] [PMID]

[13] Overend TJ, Paterson DH, Cunningham DA. The effect of interval and continuous training on the aerobic parameters. Can J Sport Sci. 1992; 17(2):129-34. [PMID]

[14] Brandon L. Physiological factors associated with middle distance running performance. Sports Med. 1995; 19(4):268-77. [DOI:10.2165/00007256-199519040-00004] [PMID]

[15] Hong A-R, Hong S-M, Shin Y-A. Effects of resistance training on muscle strength, endurance, and motor unit according to ciliary neurotrophic factor polymorphism in male college students. J Sports Sci Med. 2014 13(3):680-8. [PMCID]
[16] Assuncao AR, Bottaro M, Ferreira-Junior JB, Izquierdo M, Cadore $E L$, Gentil P. The chronic effects of low-and high-intensity resistance training on muscular fitness in adolescents. PloS One. 2016 11(8) :e0160650. [DOI:10.1371/journal.pone.0160650] [PMID] [PMCID]

[17] López-Segovia M, Andrés JMP, González-Badillo JJ. Effect of 4 months of training on aerobic power, strength, and acceleration in two under-19 soccer teams. J Strength Cond Res. 2010 24(10):2705-14. [DOI:10.1519/JSC.0b013e3181cc237d] [PMID]

[18] McGuigan MR, Winchester JB. The relationship between isometric and dynamic strength in college football players. J Sports Sc Med. 2008; 7(1):101-5. [PMCID] [PMID]

[19] Nourizadeh S, Mirjani M, Naserpour H. [The relationship between core stability, muscular endurance, and static balance, and shooting function in military soldiers (Persian)]. J Sport Biomech. 2019; 5(1):62-71. [DOI:10.32598/biomechanics.5.1.6]

[20] Assarzade Noushabadi M, Abedi B. [Effects of combination training on insulin resistance index and some inflammatory markers in inactive men (Persian)]. Horizon Med Sci. 2012; 18(3):95-105. http://hms.gmu.ac.ir/article-1-1302-en.htm

[21] Park S-K, Park J-H, Kwon Y-C, Yoon M-S, Kim C-S. The effect of long-term aerobic exercise on maximal oxygen consumption, left ventricular function and serum lipids in elderly women. J Physiol Anthropol Appl Human Sci. 2003; 22(1):11-7. [DOI:10.2114/ jpa.22.11] [PMID]

[22] Julius S. Transition from high cardiac output to elevated vascular resistance in hypertension. Am Heart J. 1988; 116(2):600-6. [DOI:10.1016/0002-8703(88)90557-1]

[23] Axelsson M, Nilsson S. Blood pressure control during exercise in the Atlantic cod, Gadus morhua. J Exp Biol. 1986; 126:225-36. [PMID]

[24] Holviala J, Kraemer WJ, Sillanpää E, Karppinen H, Avela J, Kauhanen $A$, et al. Effects of strength, endurance and combined training on muscle strength, walking speed and dynamic balance in aging men. Eur J Appl Physiol. 2012; 112(4):1335-47. [DOI:10.1007/ s00421-011-2089-7] [PMID]

[25] Khataei T, Harding AMS, Janahmadi M, El-Geneidy M, AghaAlinejad $\mathrm{H}$, Rajabi $\mathrm{H}$, et al. ASICs are required for immediate exercise-induced muscle pain and are downregulated in sensory neurons by exercise training. J Appl Physiol. 2020; 129(1):17-26 [DOI:10.1152/japplphysiol.00033.2020] [PMID]

[26] Mackała K, Kurzaj M, Okrzymowska P, Stodółka J, Coh M, RożekPiechura K. The effect of respiratory muscle training on the pulmonary function, lung ventilation, and endurance performance of young soccer players. Int J Environ Res Public Health. 2020; 17(1):234. [DOI:10.3390/ijerph17010234] [PMID] [PMCID]

[27] Nazari Pirdosti S, Ramezani A, Lotfi G. [The effect of 8-weeks an aerobic and anaerobic training on the physical abilities of 12-14 years boys (Persian)]. J Phys Educ. 2014; 3(1):19-28. http://jtpe. sru.ac.ir/article_264_en.htm

[28] Macpherson REK, Hazell TJ, Olver TD, Paterson DH, Lemon PWR. Run sprint interval training improves aerobic performance but not maximal cardiac output. Med Sci Sports Exerc. 2011; 43(1):115 22. [DOI:10.1249/MSS.0b013e3181e5eacd] [PMID]

[29] Hazell TJ, MacPherson REK, Gravelle BMR, Lemon PWR. 10 or 30-s sprint interval training bouts enhance both aerobic and anaerobic performance. Eur J Appl Physiol. 2010; 110(1):153-60. [DOI:10.1007/s00421-010-1474-y] [PMID] 
[30] Takarda Y, Ishii N. Effects of low-intensity resistance exercise with short interset rest period on muscular function in middle-aged women. J Strength Cond Res. 2002; 16(1):123-8. [DOI:10.1519/00124278-200202000-00019]

[31] Hoff J, Helgerud J. Endurance and strength training for soccer players. Sports Med. 2004; 34(3):165-80. [DOI:10.2165/00007256-20043403000003] [PMID]

[32] Helgerud J, Rodas G, Kemi OJ, Hoff J. Strength and endurance in elite football players. Int J Sports Med. 2011; 32(9):677-82. [DOI:10.1055/s-0031-1275742] [PMID]

[33] Sundberg CJ. Exercise and training during graded leg ischaemia in healthy man with special reference to effects on skeletal muscle. Acta Physiol Scand Suppl. 1994; 615:1-50. [PMID] 
